Article

\title{
Temporal and Spatial Regulation of $m i R-320$ in the Uterus during Embryo Implantation in the Rat
}

\author{
Hong-Fei Xia ${ }^{1}$, Xiao-Hua Jin ${ }^{1}$, Pei-Pei Song ${ }^{1,2}$, Yi Cui ${ }^{1,2}$, Chun-Mei Liu ${ }^{1}$ and Xu Ma ${ }^{1{ }^{1} *}$ \\ 1 Reproductive and Genetic Center, National Research Institute for Family Planning, Beijing \\ 100081, China; E-Mails: hongfeixia@yahoo.com.cn (H.-F.X.); jxh969696@yahoo.com.cn (X.-H.J.); \\ songqi_3587@163.com (P.-P.S.); cuiyi_6@hotmail.com (Y.C.); minnieliu@sohu.com (C.-M.L.) \\ Graduate School, Peking Union Medical College, Beijing 100005, China \\ * Author to whom correspondence should be addressed; E-Mail: genetic@263.net.cn; \\ Tel.: +86-10-62176870; Fax: +86-10-62179059.
}

Received: 2 December 2009 / Accepted: 10 February 2010 / Published: 11 February 2010

\begin{abstract}
The implantation process is complex, requiring reciprocal interactions between implantation-competent blastocysts and the receptive uterus. There were reports to show that some microRNAs (miRNAs) may play a key role during embryo implantation in mouse. However, the miR-320 expression profiles in the rat uterus during peri-implantation are unknown. In the present study, we found that the expression level of $m i R-320$ was lower on day 5 of gestation (g.d. 5) in rats than g.d.3 and g.d.4 and restored gradually from g.d.6. MiR-320 was specifically localized in glandular and luminal epithelia and decidua. The expression of $m i R-320$ was not significantly different in the pseudopregnant uterus and decreased in the uteri of rats subjected to activation of delayed implantation. Artificial decidualization and treatment with progesterone increased the miR-320 expression. Thus, $m i R-320$ was differentially expressed in the rat uterus during implantation. The expression level was affected by active blastocysts and decidualization during the window of implantation. Steroid hormones, progesterone stimulated miR-320 expression.
\end{abstract}

Keywords: miR-320; embryo implantation; uterus; rat; hormone; pregnancy 


\section{Introduction}

Implantation is a highly coordinated sequence of events that begins with the attachment of an embryo to the uterine luminal epithelium and ultimately results in formation of the placenta. Implantation of the embryo to the uterine wall is regulated by various factors, for example, hormones $[1,2]$, cytokines [3,4], growth factor [5,6], etc. Although a growing list of molecules are known to be involved in the implantation process, specifically, mechanisms associated with the onset of uterine receptivity and embryo implantation remains to be determined.

MicroRNAs (miRNAs) are small noncoding RNAs whose function as modulators of gene expression is crucial for proper control of cell growth [7,8] and are known to participate in mouse embryo implantation [9,10]. Mouse mir-320 was predicted [11] based on homology to a cloned human miRNA (MI0000542) [12] and cloned from mouse oocytes and testes [13]. The human miR-320 sequence was originally cloned from the normal mucosa-derived population of human[12] and rat miR-320 sequence was firstly found from large-scale cloning studies [14]. MiR-320 expression level was down-regulated in primary breast cancer (BC) [15], and inhibited HL-60 cell proliferation by targets transferrin receptor 1 (CD71) [16]. This implied that miR-320 may play an important role in the development of cancer. Embryo implantation shares similar phenomena and mechanisms with tumor invasion [17]. However, up to now, there is no information in the literature about how such miRNAs act on the rat uterus during embryo implantation.

Here, we report the expression pattern of $m i R-320$ in the uterus during embryo implantation in the rat. We studied the effect of pseudopregnancy, artificial decidualization and activation of delayed implantation on the expression of $m i R$-320. In addition, we also tested the effect of steroid hormones on miR-320 expression.

\section{Results and Discussion}

\subsection{Differential expression of miR-320 in the rat uterus during the peri-implantation period}

To study the role of miR-320 in embryo implantation, we first examined its temporal and spatial distribution in the uterus during the peri-implantation period in rat. Northern blot analysis showed that the expression level of miR-320 was lower on g.d. 5 in rats than g.d. 3 and g.d. 4 ( $<<0.05)$, and restored gradually from g.d. 6, and was higher on g.d. 8 and g.d. 9 than g.d. 5 (p < 0.05) (Figure 1 A). The in situ hybridization results showed that the $m i R-320$ was mainly located in the glandular, luminal epithelia and stroma on g.d. 3 and g.d. 4 (Figure $1 \mathrm{~B} a$ and $b$ ). This suggested that it might participate in uterine epithelial and endometrial remodeling in preparing it to receive implanting blastocysts. On g.d. 5, the miR-320 signal mainly appeared in the glandular epithelia and weak in the luminal epithelia and stroma (Figure 1 B c). A miR-320 signal was found in the deciduas, glandular and luminal epithelia on g.d. 6 (Figure $1 \mathrm{~B} d$ ) and was strengthened in deciduas from g.d. 7 (Figure $1 \mathrm{~B} e$ ). In rat, blastocysts entered into uterus on g.d. 5 and began to implant from g.d. 6. In this study, the implantation sites and interimplantation sites in uterus were not examined independently and whole uterus was collected to perform the experiment of Northern blot and in situ hybridization after embryo implantation. The in situ results were from interimplantation uterine regions on g.d. 6. The above- 
mentioned observations suggested that the presence of blastocysts may reduce the expression of $m i R$ 320 in the uterine luminal epithelia and stroma and decidualization may induce the expression of $m i R$ 320 in pregnant rats.

Figure 1. Changes in uterine miR-320 expression during early pregnancy.

A
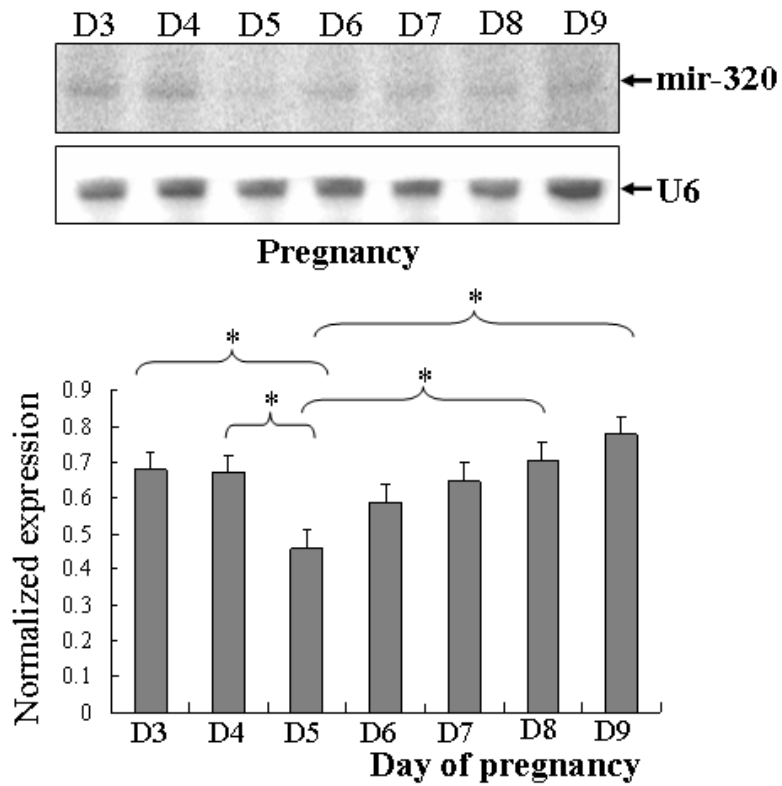

B
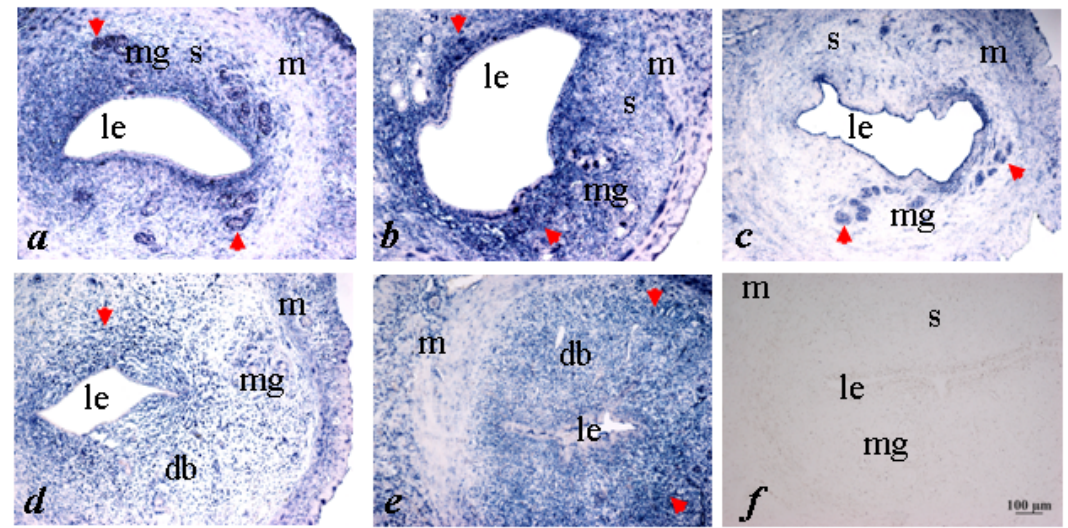

(A) Northern blot analysis of uterine miR-320 expression during days 3-9 of pregnancy. Hybridization was done with a ${ }^{32} \mathrm{P}$-labeled probe for $m i R-320$ and $U 6$. The black curve represents the optical densities of the signals quantified by densitometric analysis and represented as a ratio of $m i R-320$ to $U 6$ intensity to normalize for gel loading and transfer. (B) In situ localization of $m i R$ 320 in the rat uterus during early pregnancy. Sections of the uterus from days $3(a), 4(b), 5(c)$, $6(d), 7(e)$ of pregnancy were subjected to in situ hybridization using DIG-labeled LNA probes specific to mir-320. Staining was developed using BCIP/NBT: blue staining indicates a hybridization signal. To evaluate the specificity of the probe, negative control staining was performed by substituting as DIG-labeled LNA-scrambled probe for the DIG-labeled LNA-miR320 probe $(f)$. The scale bar indicated a distance of $100 \mu \mathrm{M}$. Key: m, myometrium; mg, maternal gland; s, stroma; le, luminal epithelium; db, decidua basalis. 
Figure 2. Changes in uterine miR-320 expression during pseudopregnancy.

$\mathbf{A}$

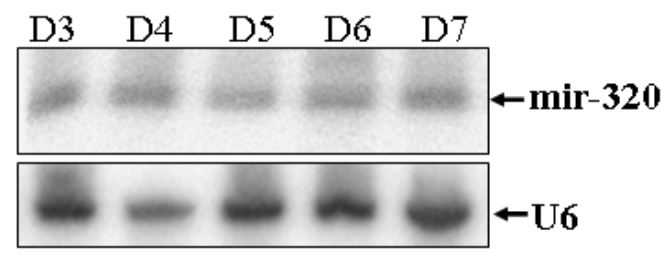

Day of pseudopregnancy

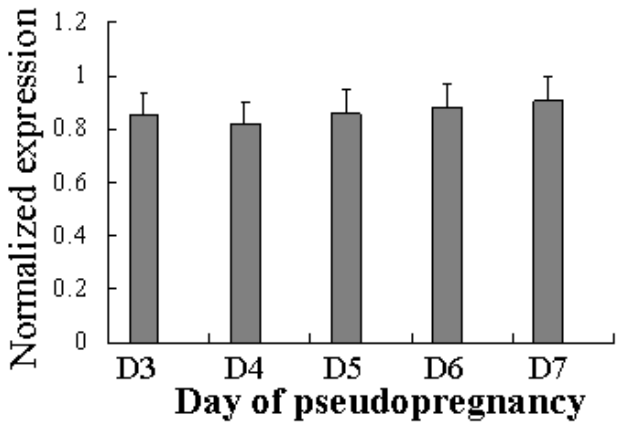

B
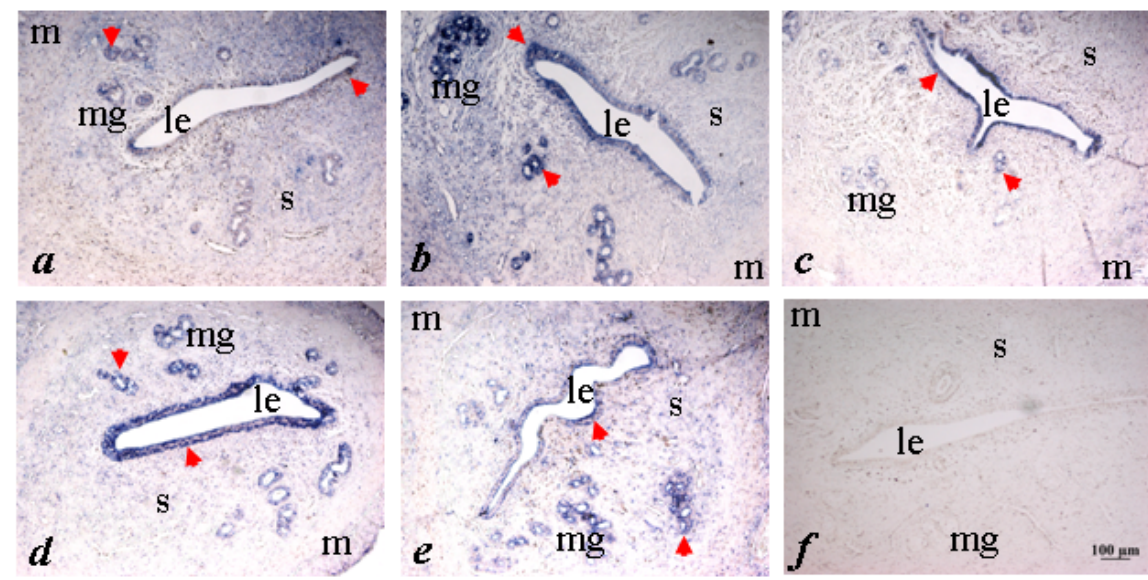

(A) Northern blot analysis of uterine miR-320 expression during days 3-7 of pseudopregnancy. Hybridization was done with a ${ }^{32} \mathrm{P}$-labeled probe for $m i R-320$ and $U 6$. The black curve represents the optical densities of the signals quantified by densitometric analysis and represented as a ratio of $m i R-320$ to U6 intensity to normalize for gel loading and transfer. (B) In situ localization of $m i R$ 320 in the rat uterus during pseudopregnancy. Sections of the uterus from days 3 (a) 4 (b), 5 (c), $6(d), 7$ (e) of pseudopregnancy were subjected to in situ hybridization using DIG-labeled LNA probes specific to mir-320. Staining was developed using BCIP/NBT: blue staining indicates a hybridization signal. To evaluate the specificity of the probe, negative control staining was performed by substituting as DIG-labeled LNA-scrambled probe for the DIG-labeled LNA-miR320 probe $(f)$. The scale bar indicated a distance of $100 \mu \mathrm{M}$. Key: m, myometrium; m, maternal gland; s, stroma; le, luminal epithelium; db, decidua basalis.

\subsection{Pseudopregnancy did not change the expression of miR-320}

To see whether the miR-320 expression was dependent upon the presence of embryos, uterine tissues were subjected to Northern blot and in situ hybridization analysis (Figure 2 A). The expression 
level of mir-320 detected by Northern blot was not significantly different in uterus during days 3-7 of pseudopregnancy. In situ hybridization showed that the miR-320 signal was mainly found in uterine glandular and luminal epithelia during days 3-7 of pseudopregnancy (Figure 2 B). The expression level of miR-320 is higher during g.d.3-4 than g.d. 5-7 in the stroma. These results suggested that the miR320 expression is not dependent upon the presence of embryos in pregnant rats.

\subsection{Delayed implantation inhibited the expression of miR-320}

To test whether the miR-320 expression was dependent upon embryo implantation status, a delayed implantation model was used for Northern blot and in situ hybridization analyses. Northern blot showed a high level of miR-320 in the uterus under delayed implantation conditions, but it decreased significantly after implantation was activated with estrogen treatment $(P<0.05$; Figure $3 \mathrm{~A})$. In situ hybridization showed that a strong signal appeared in the uterine glandular and luminal epithelia and weak in stroma during delayed implantation (Figure $3 \mathrm{~B} a, b$ ). After implantation was activated by estrogen treatment and the embryos had implanted, there was weak miR-320 expression in the stroma, glandular and luminal epithelia (Figure $3 \mathrm{~B} \mathrm{c}$ ), suggesting that down-regulation of $m i R-320$ expression was dependent upon the presence of viable implanting blastocysts.

Figure 3. The expression of $m i R-320$ in the uterus of delayed implantation.

A

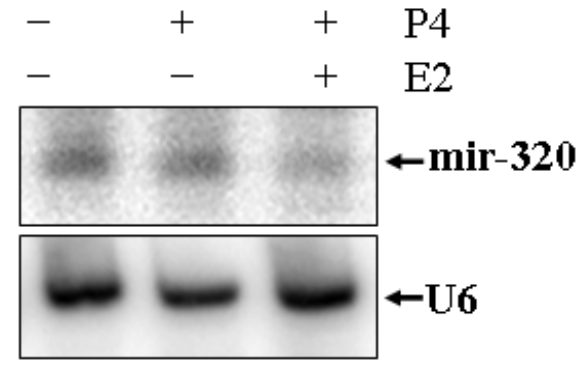

Delayed-implantation

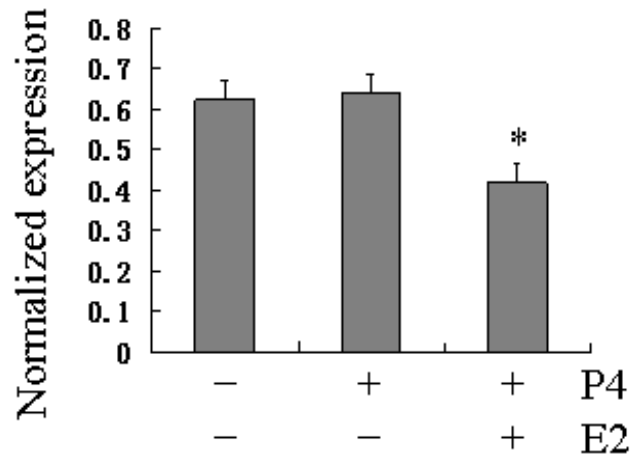


Figure 3. Cont.

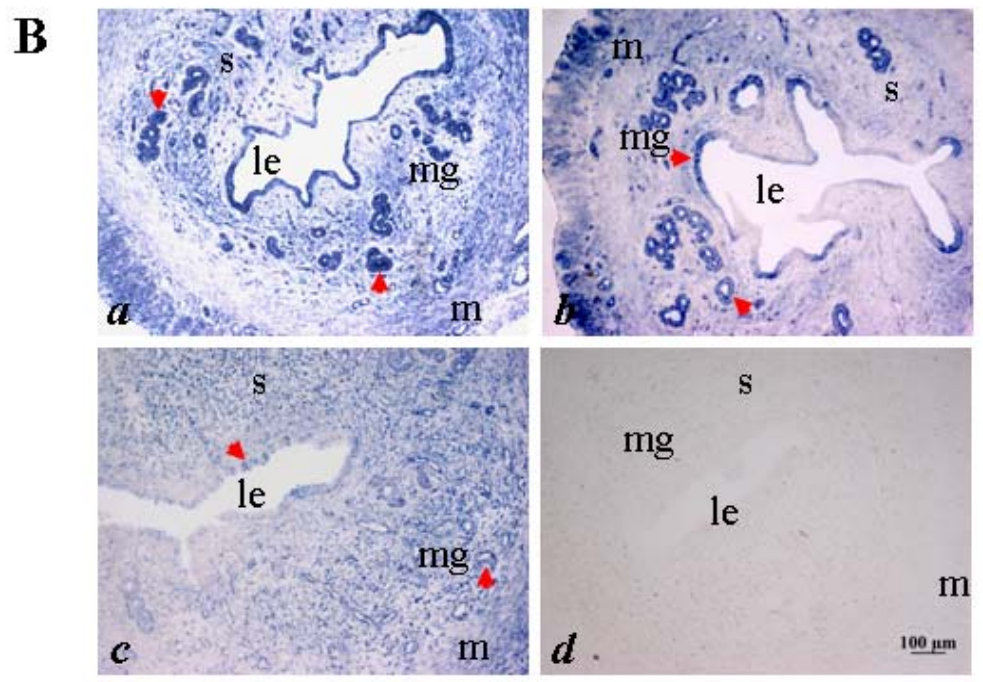

(A) Northern blot analysis of uterine miR-320 expression in a model of delayed implantation. Hybridization was done with a ${ }^{32} \mathrm{P}$-labeled probe for $m i R-320$ and $U 6$. The black curve represents the optical densities of the signals quantified by densitometric analysis and represented as a ratio of miR-320 to U6 intensity to normalize for gel loading and transfer. (B) Sections of the uterus from delayed implantation ( $a$ and $b$ ) and activation of delayed implantation (c) were subjected to in situ hybridization using DIG-labeled LNA probes specific to mir-320. To evaluate the specificity of the probe, negative control staining was performed by substituting as DIG-labeled LNA-scrambled probe for the DIG-labeled LNA-miR-320 probe (d). The scale bar indicated a distance of $100 \mu \mathrm{M}$. Key: m, myometrium; m, maternal gland; s, stroma; le, luminal epithelium; db, decidua basalis.

\subsection{Experimentally induced decidualization increases the expression of miR-320}

To test whether miR-320 expression was regulated by decidualization, a model of experimentally induced decidualization was used for Northern blot and in situ hybridization analyses. The expression level of miR-320 in the decidualized uterus was evidently higher than in the nonstimulated uterus on day 7 of pseudopregnancy (Figure 4 A). In situ hybridization showed strong staining in glandular and luminal epithelia in the control uterine horn on day 7 of pseudopregnancy (Figure 4 B a). However, in the oil-infused uterus, strong signals were detected in decidua but staining was weak in the luminal epithelium (Figure $4 \mathrm{~B} b$ ). This indicated that artificial decidualization promoted the expression of $m i R-320$ and further emphasize the importance of decidualization in regulating the dynamics of $m i R-$ 320 expression in the uterus during the window of implantation.

\subsection{Progesterone enhances the miR-320 expression}

Ovarian progesterone and estrogen are the principal hormones that direct uterine receptivity, embryo implantation and the maintenance of pregnancy in all mammals studied, and are essential for implantation in mice and rats $[19,20]$. 
Figure 4. The expression of $m i R-320$ in the uterus of artificial decidualization.

A

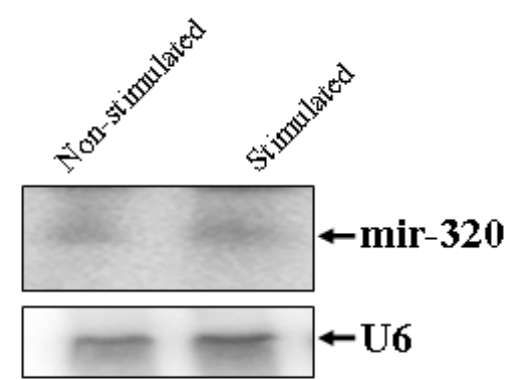

Artificial decidualization

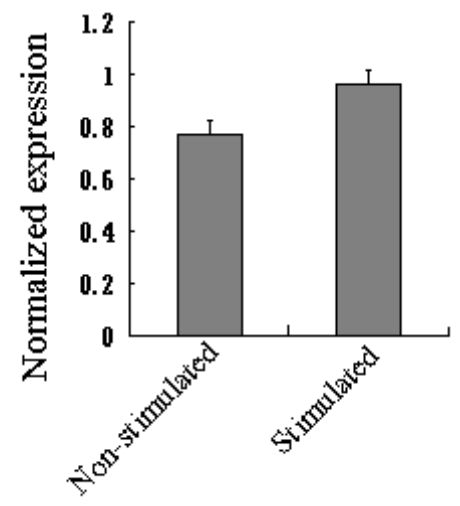

B
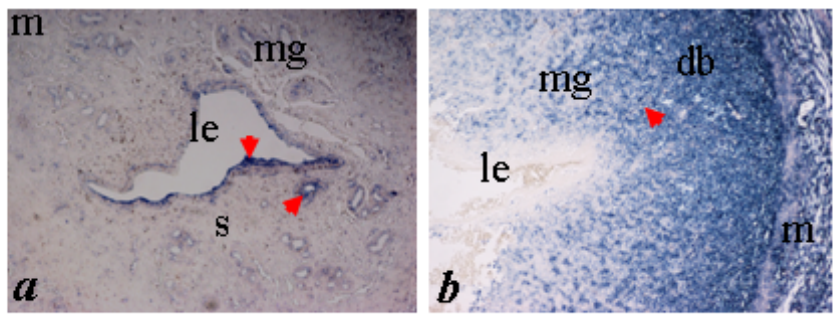

m

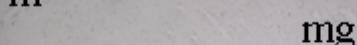

le

$\mathrm{s}$

c

(A) Northern blot analysis of uterine miR-320 expression in a model of artificial decidualization. Hybridization was done with a ${ }^{32} \mathrm{P}$-labeled probe for $m i R-320$ and $U 6$. The black curve represents the optical densities of the signals quantified by densitometric analysis and represented as a ratio of $m i R-320$ to U6 intensity to normalize for gel loading and transfer. (B) In situ localization of miR320 in the uterus of rats showing artificial decidualization. Artificial decidualization was stimulated by infusing $100 \mu \mathrm{L}$ sesame oil into the lumen of one of the uterine horns $(b)$. The contralateral uterine horn, which was not infused with oil, served as a nonstimulated control (a). Sections of the uterus were subjected to in situ hybridization using DIG-labeled LNA probes specific to mir-320. The scale bar indicated a distance of $100 \mu \mathrm{M}$. To evaluate the specificity of the probe, negative control staining was performed by substituting as DIG-labeled LNA-scrambled probe for the DIGlabeled LNA-miR-320 probe (c). Key: m, myometrium; m, maternal gland; s, stroma; le, luminal epithelium; db, decidua basalis.

In order to test the effect of steroid hormones on the miR-320 expression under physiological condition, Northern blot was performed to examine whether the miR-320 expression was regulated by steroid hormones. A low level of miR-320 expression was detected in the ovariectomized rat uterus. 
However, treatment with progesterone significantly increased miR-320 expression $(P<0.01)$ and estradiol-17 $\beta$ did not visibly affect the expression level of miR-320. The miR-320 expression was slightly increased by combination of both (Figure 5). All these facts indicated that progesterone can promote $m i R-320$ expression under physiological condition.

Figure 5. The effect of steroid hormones on uterine miR-320 expression. The effect of steroid hormones on uterine miR-320 expression steroid hormones on uterine miR-320 expression was detected by Northern blot. Hybridization was done with a ${ }^{32} \mathrm{P}$-labeled probe for $m i R-320$ and U6. The black curve represents the optical densities of the signals quantified by densitometric analysis and represented as a ratio of miR-320 to U6 intensity to normalize for gel loading and transfer.
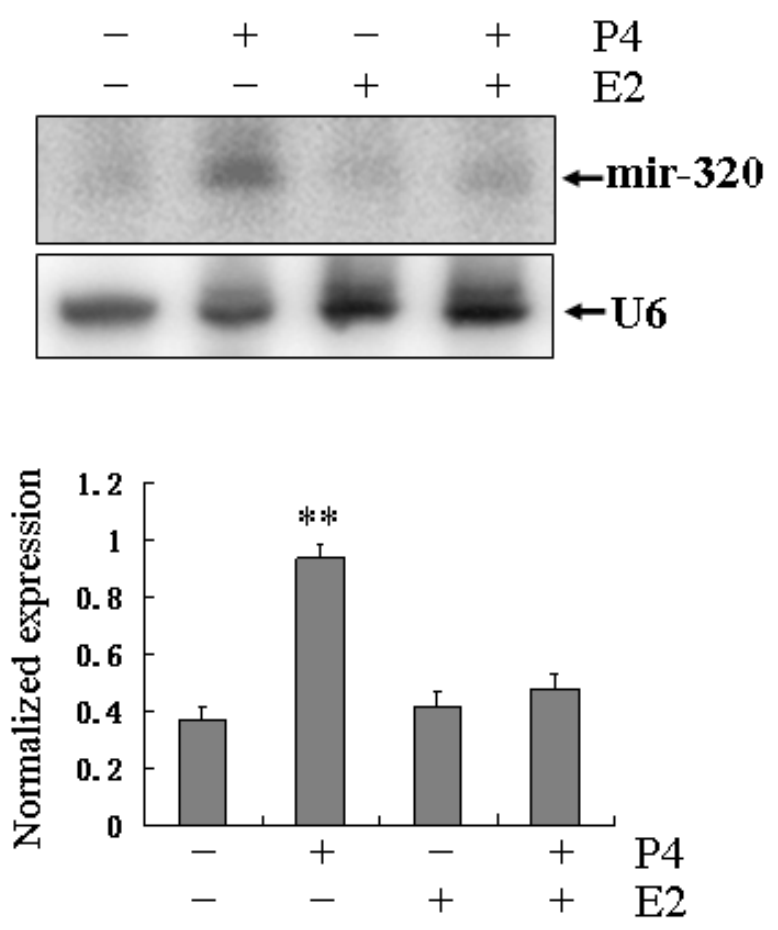

Progesterone $\left(\mathrm{P}_{4}\right)$ is essential for the development of endometrial receptivity for blastocyst implantation under pregnant condition [28,29] and play an important role on the maintenance of female endocrine homeostasis by inhibiting the secretion of gonadotropin in hypothalamus. Endometrial receptivity for embryo implantation in the rat occurred on day 5 of pregnancy. The expression of $m i R-320$ is decreased on g.d.5, but its expression was increased by progesterone under physiological condition. These results implied that the low expression of miR-320 may be benefit to formation of endometrial receptivity under pregnant condition and the high expression of miR-320 may be in favor of the maintenance of female endocrine homeostasis. In addition, there are striking similarities between the behavior of invasive placental cells and that of invasive cancer cells. Dysregulated expression of the miR-320 in many tumor $[15,16]$ was strongly associated with tumor development, and miR-320 expression level was down-regulated greater than two fold in primary breast cancer (BC) [15]. These results imply that the action of progesterone on miR-320 expression might inhibit the excess invasion of cells from the uterus and trophectoderm. 


\section{Experimental Section}

\subsection{Experimental animals and protocols}

Sexually mature, healthy female Sprague Dawley rats (220-260 g body weight) were purchased from the Laboratory Animal Center of the Academy of Military Medical Sciences (Beijing, PR China). The rats were housed in a temperature- and humidity-controlled room with a 12/12 h light/dark cycle. All animal procedures were approved by the Institutional Animals Care and Use Committee of the National Research Institute for Family Planning. The rats were caged overnight with fertile males of the same strain. The presence of a vaginal plug or sperm was considered to be day 1 of pregnancy (g.d.1). The whole uterus was collected from g.d. 3-5 rats. When embryos implant and placentae form, placentae were carefully peeled from dissected uterine horns and whole uterus including the peritoneum, myometrium and maternal decidua was collected from g.d. 6-9 rat. Divided uteri were respectively frozen in Eppendorf tubes and stored at $-80{ }^{\circ} \mathrm{C}$ until processing for RNA extraction. Whole uteri or undivided uteri and placentae were fixed in $4 \%$ paraformaldehyde (PFA) solution (Sigma-Aldrich, St. Louis, MO, USA) in $0.1 \mathrm{M}$ phosphate buffer $\left(\mathrm{pH} 7.4,4{ }^{\circ} \mathrm{C}\right.$ ) for in situ hybridization analysis.

Pseudopregnancy was induced by caging adult females with vasectomized males, and mating was confirmed by checking for a vaginal plug (day 1 of pseudopregnancy). The whole uteri were collected from days 3-7 of pseudopregnancy. On day 5 of pseudopregnancy, when the uteri were optimally sensitized to deciduogenic stimuli, $100 \mu \mathrm{L}$ olive oil (Sigma-Aldrich) was infused into the lumen of one of the uterine horns to induce artificial decidualization. The contralateral uterine horn, which was not infused with oil, served as a control. At day 7 of pseudopregnancy, the rats were sacrificed and the uterine horns were isolated.

To induce delayed implantation, the pregnant rats on g.d. 4 were ovariectomized. Progesterone ( $5 \mathrm{mg} /$ rat, s.c.; Sigma-Aldrich) was injected to maintain delayed implantation from g.d. 5-7. The progesterone-primed delayed-implantation rats were treated with estradiol-17 $\beta$ ( $0.5 \mu \mathrm{g} / \mathrm{rat}$; SigmaAldrich) to terminate delayed implantation. The rats were sacrificed by stunning and cervical dislocation to collect uteri $24 \mathrm{~h}$ after estrogen treatment. The implantation sites were also identified by i.v. injection of Chicago blue solution (Sigma-Aldrich). Delayed implantation was confirmed by flushing the blastocysts from the uterus.

To test the effects of steroid hormones on miR-320 expression, rats were treated with hormones starting 2 weeks after they were ovariectomized. The ovariectomized rats were treated with an injection of estradiol-17 $\beta$ ( $1 \mu \mathrm{g} / \mathrm{rat})$ or progesterone $(10 \mathrm{mg} / \mathrm{rat})$ at intervals of $24 \mathrm{~h}$ for $3 \mathrm{~d}$. All steroids were dissolved in olive oil and injected subcutaneously. Controls received the vehicle only $(0.1 \mathrm{~mL} / \mathrm{rat})$.

\subsection{Northern blot analysis}

Northern blot analysis of miRNAs was performed as described previously [22]. Briefly, total RNA was isolated from the uteri of rats with TRIzol reagent (Invitrogen, Carlsbad, CA, USA). Aliquots of $40 \mu \mathrm{g}$ of total RNA per sample were subjected to electrophoresis on a $15 \%$ urea-PAGE gel and 
transferred to a nylon membrane (Hybond N+; Amersham Pharmacia Biotech, St Albans, Hertford, UK). After being UV cross-linked and baked at $50{ }^{\circ} \mathrm{C}$ for $30 \mathrm{~min}$, the membrane was prehybridized at $42{ }^{\circ} \mathrm{C}$ for $4 \mathrm{~h}$ and then hybridized with ${ }^{32} \mathrm{P}$-labeled probes at $40{ }^{\circ} \mathrm{C}$ overnight. Membranes were washed and exposed to PhosphorImager screens (GE Healthcare Bio-Sciences Corp., Piscataway, NJ, USA). The bands were analyzed using the Quantity One software (Bio-Rad, Hercules, CA, USA). All experiments were repeated at least three times.

\subsection{In situ hybridization of miR-320 with DIG-labeled LNA probes}

In situ hybridization of miRNAs with DIG-labeled LNA probes was performed as described previously [23]. Briefly, sections of uterus $(5 \mu \mathrm{m})$ were treated with proteinase $\mathrm{K}(20 \mathrm{~g} / \mathrm{mL})$ for $15 \mathrm{~min}$ and refixed in 4\% PFA for $15 \mathrm{~min}$. After acetylation with $0.25 \%$ acetic anhydride in $0.1 \mathrm{M}$ triethanolamine ( $\mathrm{pH} \mathrm{8.0)}$ for $10 \mathrm{~min}$, sections were prehybridized with hybridization buffer (Roche, Mannheim, Germany) at $40{ }^{\circ} \mathrm{C}$ for $2 \mathrm{~h}$ and then hybridized with digoxigenin (DIG)-labeled LNA-miR320 probe (LNA-miR-320 sequence: $5^{\prime}-\mathrm{DIG}-\mathrm{ttCgcCctCtCaAcCcAgCtttt-3}$ ) at $40{ }^{\circ} \mathrm{C}$ overnight. The cells were then incubated in buffer containing anti-DIG-antibody for $2 \mathrm{~h}$ at $37^{\circ} \mathrm{C}$ and stained with 5bromo-4-chloro-3-indolyl phosphate (BCIP; Promega, Madison, WI, USA) and p-nitroblue tetrazolium chloride (NBT; Promega, Madison, WI, USA). The cells and sections were hybridized with a DIGlabeled LNA-scrambled probe (LNA-scrambled sequences: 5'-caTtaAtgTcGgaCaaCtcAat-3') as a negative control [24]. Samples were viewed with an Eclipse 80i microscope (Nikon, Tokyo, Japan).

\subsection{Statistical analysis}

There were at least three rats in each treatment group. The results of Northern blot and in situ hybridization were repeated three times. All values are reported as the mean $\pm \mathrm{SE}$. Statistical analysis was performed using one-way ANOVA. When significant effects of treatments were indicated, the Student-Newman-Keuls multiple range test was applied using SPSS version 13.0 (SPSS Inc., Chicago, IL, USA). $P<0.05$ was considered statistically significant.

\section{Conclusions}

In conclusion, we found that the miRNA miR-320 could be detected differentially in the rat uterus during the peri-implantation period. The results obtained from our models of pseudopregnancy, artificial decidualization and delayed implantation imply an important role for implanting blastocysts and decidualization in the temporal and spatial changes of $m i R-320$ expression in the uterus during the window of implantation. In addition, this expression of miR-320 was regulated by progesterone. Collectively, these findings will help us gain a better understanding of the role of mir-320 during pregnancy and provide a foundation for futher experimental studies on mechanisms mechanisms associated with the onset of uterine receptivity and embryo implantation. 


\section{Acknowledgements}

This work was supported by the Natural Science Foundation of China (No. 30800396) and the National Basic Research Program of China (No. 2007CB511905).

\section{References}

1. Venners, S.A.; Liu, X.; Perry, M.J.; Korrick, S.A.; Li, Z.; Yang, F.; Yang, J.; Lasley, B.L.; Xu, $\mathrm{X}$; Wang, X. Urinary estrogen and progesterone metabolite concentrations in menstrual cycles of fertile women with non-conception, early pregnancy loss or clinical pregnancy. Hum. Reprod. 2006, 21, 2272-2280.

2. Yoshinaga, K. Review of factors essential for blastocyst implantation for their modulating effects on the maternal immune system. Semin. Cell. Dev. Biol. 2008, 19, 161-169.

3. Sun, Q.H.; Peng, J.P.; Xia, H.F.; Yang, Y.; Liu, M.L. Effect on expression of RT1-A and RT1DM molecules of treatment with interferon-gamma at the maternal--fetal interface of pregnant rats. Hum. Reprod. 2005, 20, 2639-2647.

4. Xia, H.F.; Sun, Q.H.; Peng, J.P. Effect of interferon-gamma treatment on the expression of interleukin-1beta at the maternal-fetal interface of pregnant rats. Reprod. Fertil. Dev. 2007, 19, $510-519$.

5. Sohlström, A.; Katsman, A.; Kind, K.L.; Roberts, C.T.; Owens, P.C.; Robinson, J.S.; Owens, J.A. Food restriction alters pregnancy-associated changes in IGF and IGFBP in the guinea pig. Am. J. Physiol. 1998, 274, E410-E416.

6. Roberts, C.T.; Kind, K.L.; Earl, R.A.; Grant, P.A.; Robinson, J.S.; Sohlstrom, A.; Owens, P.C.; Owens, J.A. Circulating insulin-like growth factor (IGF)-I and IGF binding proteins -1 and -3 and placental development in the guinea-pig. Placenta 2002, 23, 763-770.

7. Sampson, V.B.; Rong, N.H.; Han, J.; Yang, Q.; Aris, V.; Soteropoulos, P.; Petrelli, N.J.; Dunn, S.P.; Krueger, L.J. MicroRNA let-7a down-regulates MYC and reverts MYC-induced growth in Burkitt lymphoma cells. Cancer Res. 2007, 67, 9762-9770.

8. Abdelmohsen, K.; Srikantan, S.; Kuwano, Y.; Gorospe, M miR-519 reduces cell proliferation by lowering RNA-binding protein HuR levels. Proc. Natl. Acad. Sci. USA 2008, 105, 20297-20302.

9. Chakrabarty, A.; Tranguch, S.; Daikoku, T.; Jensen, K.; Furneaux, H.; Dey, S.K. MicroRNA regulation of cyclooxygenase-2 during embryo implantation. Proc. Natl. Acad. Sci. USA 2007, 104, 15144-15149.

10. Hu, S.J.; Ren, G.; Liu, J.L.; Zhao, Z.A.; Yu, Y.S.; Su, R.W.; Ma, X.H.; Ni, H.; Lei, W.; Yang, Z.M. MicroRNA expression and regulation in mouse uterus during embryo implantation. J. Biol. Chem. 2008, 283, 23473-23484.

11. Weber, M.J. New human and mouse microRNA genes found by homology search. FEBS J. 2005, 272, 59-73.

12. Michael, M.Z.; O' Connor, S.M.; van Holst Pellekaan, N.G.; Young, G.P.; James, R.J. Reduced accumulation of specific microRNAs in colorectal neoplasia. Mol. Cancer. Res. 2003, 1, 882-891.

13. Watanabe, T.; Takeda, A.; Tsukiyama, T.; Mise, K.; Okuno, T.; Sasaki, H.; Minami, N.; Imai, H. Identification and characterization of two novel classes of small RNAs in the mouse germline: 
Retrotransposon-derived siRNAs in oocytes and germline small RNAs in testes. Genes Dev. 2006, 20, 1732-1743.

14. Landgraf, P.; Rusu, M.; Sheridan, R.; Sewer, A.; Iovino, N.; Aravin, A.; Pfeffer, S.; Rice, A.; Kamphorst, A.O.; Landthaler, M.; Lin, C.; Socci, N.D.; Hermida, L.; Fulci, V.; Chiaretti, S.; Foà, R.; Schliwka, J.; Fuchs, U.; Novosel, A.; Müller, R.U.; Schermer, B.; Bissels, U.; Inman, J.; Phan, Q.; Chien, M.; Weir, D.B.; Choksi, R.; De Vita, G.; Frezzetti, D.; Trompeter, H.I.; Hornung, V.; Teng, G.; Hartmann, G.; Palkovits, M.; Di Lauro, R.; Wernet, P.; Macino, G.; Rogler, C.E.; Nagle, J.W.; Ju, J.; Papavasiliou, F.N.; Benzing, T.; Lichter, P.; Tam, W.; Brownstein, M.J.; Bosio, A.; Borkhardt, A.; Russo, J.J.; Sander, C.; Zavolan, M.; Tuschl, T. A mammalian microRNA expression atlas based on small RNA library sequencing. Cell 2007, 129, 1401-1414.

15. Yan, L.X.; Huang, X.F.; Shao, Q.; Huang, M.Y.; Deng, L.; Wu, Q.L.; Zeng, Y.X.; Shao, J.Y. MicroRNA miR-21 overexpression in human breast cancer is associated with advanced clinical stage, lymph node metastasis and patient poor prognosis. RNA 2008, 14, 2348-2360.

16. Schaar, D.G.; Medina, D.J.; Moore, D.F.; Strair, R.K.; Ting, Y. MiR-320 targets transferrin receptor 1 (CD71) and inhibits cell proliferation. Exp. Hematol. 2009, 37, 245-255.

17. Murray, M.J.; Lessey, B.A. Embryo implantation and tumor metastasis: common pathways of invasion and angiogenesis. Semin. Reprod. Endocrinol. 1999, 17, 275-290.

18. Dey, S.K.; Lim, H.; Das, S.K.; Reese, J.; Paria, B.C.; Daikoku, T.; Wang, H. Molecular cues to implantation. Endocr. Rev. 2004, 25, 341-373.

19. Wang, H.; Dey, S.K. Roadmap to embryo implantation: Clues from mouse models. Nat. Rev. Genet. 2006, 7, 185-199.

20. Quinn, C.E.; Simmons, D.G.; Kennedy, T.G. Expression of Cystatin C in the rat endometrium during the peri-implantation period. Biochem. Biophys. Res. Commun. 2006, 349, 236-244.

21. Dai, B.; Cao, Y.; Liu, W.; Li, S.; Yang, Y.; Chen, D.; Duan, E. Dual roles of progesterone in embryo implantation in mouse. Endocrine 2003, 21, 123-132.

22. Zhao, J.J.; Hua, Y.J.; Sun, D.G.; Meng, X.X.; Xiao, H.S.; Ma, X. Genome-wide microRNA profiling in human fetal nervous tissues by oligonucleotide microarray. Child's Nerv. Syst. 2006, 22, 1419-1425.

23. Zhao, J.J.; Sun, D.G.; Wang, J.; Liu, S.R.; Zhang, C.Y.; Zhu, M.X.; Ma, X. Retinoic acid downregulates microRNAs to induce abnormal development of spinal cord in spina bifida rat model. Child's Nerv. Syst. 2008, 24, 485-492.

24. Chan, J.A.; Krichevsky, A.M.; Kosik, K.S. MicroRNA-21 is an antiapoptotic factor in human glioblastoma cells. Cancer Res. 2005, 65, 6029-6033.

(C) 2010 by the authors; licensee Molecular Diversity Preservation International, Basel, Switzerland. This article is an open-access article distributed under the terms and conditions of the Creative Commons Attribution license (http://creativecommons.org/licenses/by/3.0/). 